\title{
A current approach to magnetization switching
}

Sci. Adv. 4, eaat9989 (2018)

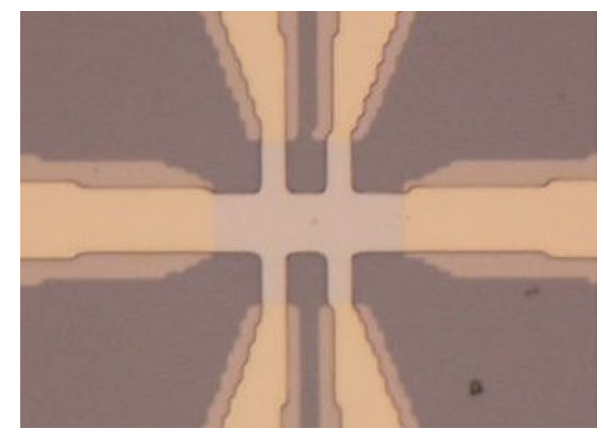

Credit: AAAS, under a Creative Commons license CC BY-NC 4.0

The electrical manipulation of magnetic properties through spin-orbit phenomena is useful for the development of magnetic memories and other spintronic devices. The orientation of magnetic domains can, in particular, be controlled by the injection of current via the Rashba-Edelstein effect, which is related to the coupling of the momentum of an electron to its spin. This current-induced switching has previously been demonstrated in ferromagnetic semiconductors and ferromagnetic metals without inversion symmetry. Ryutaro Yoshimi and colleagues have now shown that current pulses can be used to switch the magnetization direction of a ferromagnetic bulk Rashba semiconductor with broken inversion symmetry.

The researchers - who are based at the RIKEN Center for Emergent Matter Science, the University of Tokyo and Tohoku University - fabricated thin films of germanium telluride that had been doped with manganese in order to introduce ferroelectricity into the crystal structure. To probe the Rashba-Edelstein effect, they studied the Hall resistance of the $(\mathrm{Ge}, \mathrm{Mn}) \mathrm{Te}$ thin films as a function of injected current under small magnetic fields. The magnetization switching was found to be around $10 \%$ of a full magnetization reversal. Furthermore, they studied films with varying thicknesses and discovered that the switching ratio is dependent on the Fermi level position within the crystal energy bands and that it increases with higher hole density. The observation of magnetization reversal in thicker films also confirmed that the effect originates in the bulk and is not an interfacerelated effect.

Christiana Varnava

Published online: 16 January 2019

https://doi.org/10.1038/s41928-018-0201-2 